\title{
Monopod, Bipod, Tripod and Tetrapod Gold Nanocrystals
}

\author{
Sihai Chen, ${ }^{* \dagger}$ Zhong Lin Wang, ${ }^{\dagger}$ and John Ballato, ${ }^{\dagger}$ Stephen H. Foulger, ${ }^{\dagger}$ and David L. Carroll ${ }^{\S}$
}

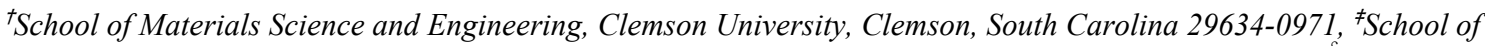
Materials Science and Engineering, Georgia Institute of Technology, Atlanta, Georgia 30332-0245, and ${ }^{\S}$ Center for Nanotechnology, Department of Physics, Wake Forest University, Winston-Salem, North Carolina 27109-7507

E-mail: chens@clemson.edu

\section{Supporting information:}

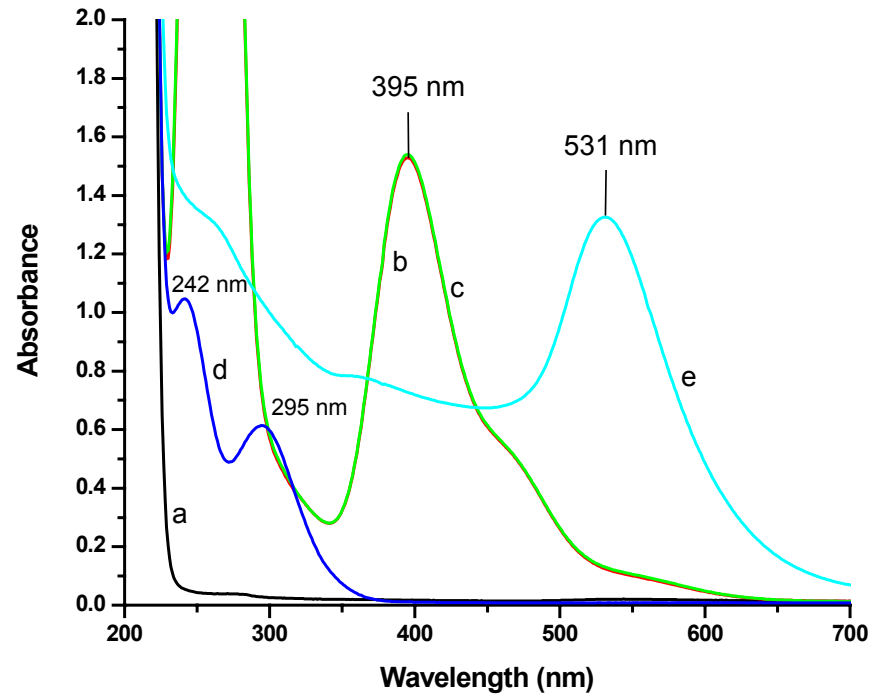

Figure 51. Absorption spectra recorded during the synthesis of gold nanocrystals. (a) $0.1 \mathrm{M} \mathrm{CTAB}$, the solution is clear, no apparent absorption was observed for wavelength larger than $240 \mathrm{~nm}$. (b) After the addition of $1.2 \mathrm{~mL}$ of $10 \mathrm{mM} \mathrm{HAuCl}_{4}$, the solution displayed a brown color with two absorption peaks at $258 \mathrm{~nm}$ and $395 \mathrm{~nm}$ (intensity ratio about 8:1), as well as a shoulder peak at $467 \mathrm{~nm}$. (c) After addition of seeds, there is no effect on the absorption spectra at this step. (d) Addition of $2.5 \mathrm{~mL} 10 \mathrm{mM}$ L-ascorbic acid, the brown color disappeared, two peaks at $242 \mathrm{~nm}$ and $295 \mathrm{~nm}$ were observed in the spectrum. (e) Final addition of $0.2 \mathrm{~mL} 1 \mathrm{M} \mathrm{NaOH}$ resulted in the formation of gold nanocrystals, which is indicated by an absorption peak located at $531 \mathrm{~nm}$ (this spectrum is recorded after two months of aging. For the time-dependent spectrum change after the addition of $\mathrm{NaOH}$, see Figure $\mathrm{S} 3$ ). 


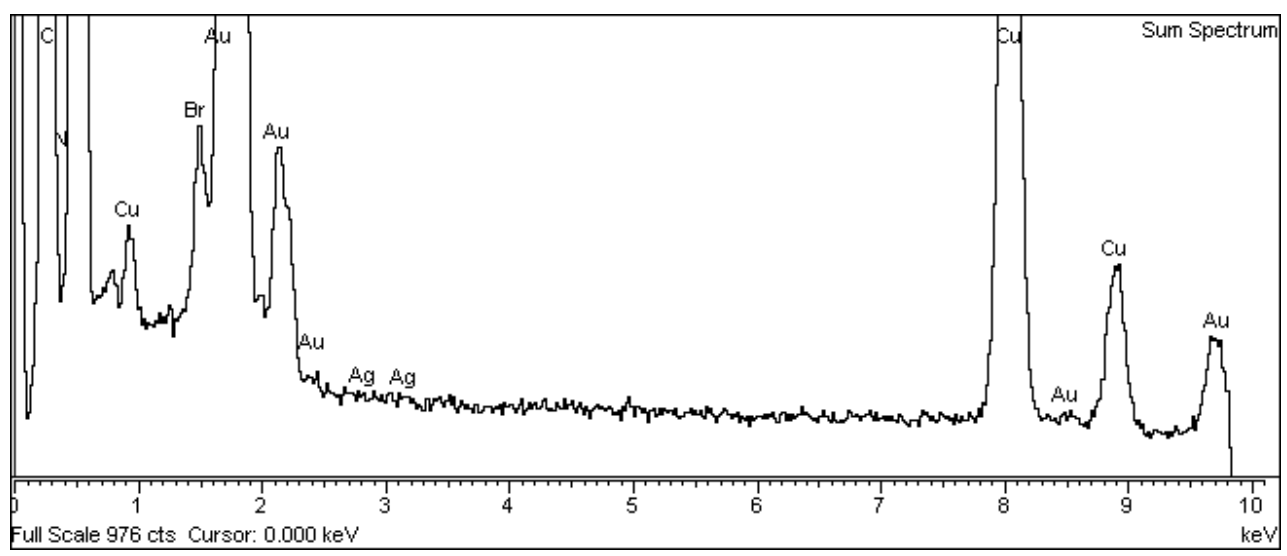

Figure S2. Element analysis result of the branched nanoparticles. The analysis is conducted with a Hitachi HD-2000 STEM thin film analysis system operated at $200 \mathrm{kV}$. The sample was prepared by dropping particle solution on Formavor coated copper mesh, and washing it with deionized water for several times to ensure removing inorganic ions or surfactants. The analyzed area is $1000 \mathrm{~nm} \times 700 \mathrm{~nm}$ with more than 200 particles randomly distributed on the substrate. It is clearly seen that gold, bromine and nitrogen are present while that of silver does not exist. The presence of copper is due to the copper mesh. The presence of bromine and nitrogen is an indication that CTAB molecules adsorb on the nanocrystal surface.

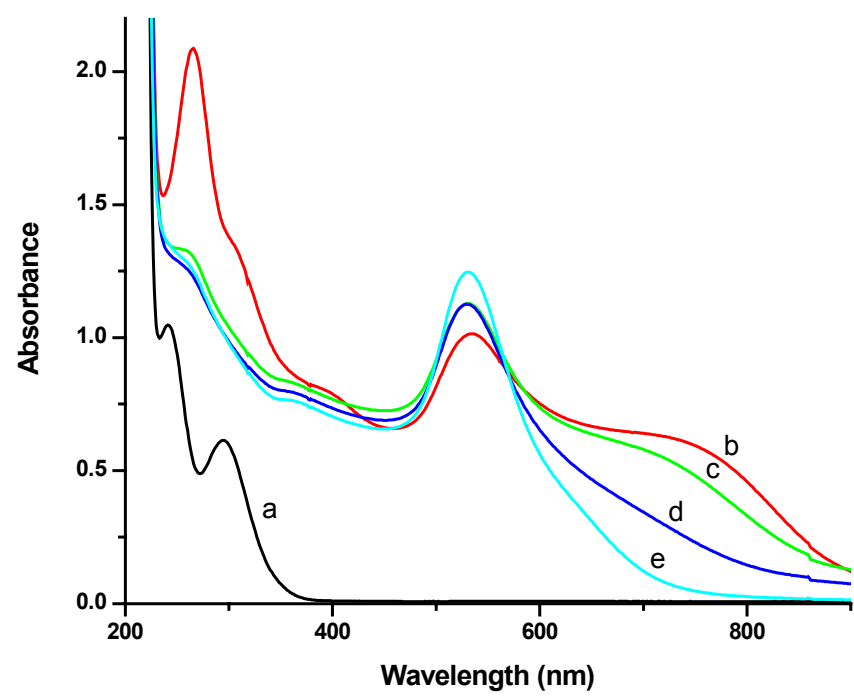

Figure S3. Absorption spectra recorded (a) before and after the addition of $\mathrm{NaOH}$ for (b) 10 min, (c) 1 hr, (d) 1 day and (e) 20 days. A shoulder peak at around $670 \mathrm{~nm}$ appeared at the initial step of particle formation, this peak gradually disappeared after 20 days of aging, reflecting the single pod toward multipod growth process. The appearance of the shoulder peak at the longer wavelength side of the main $531 \mathrm{~nm}$ peak is due to the surface plasmon band of gold corresponding to the longitudinal resonance of rod- or wire-like structures. The disappearance of this peak can be understood considering the gradual formation of multipods. See: Yu, Y.-Y.; Chang, S.-S.; Lee, C.-L.; Wang, C. R. C. J. Phys. Chem. B. 1997, 101, 6661-6664. 

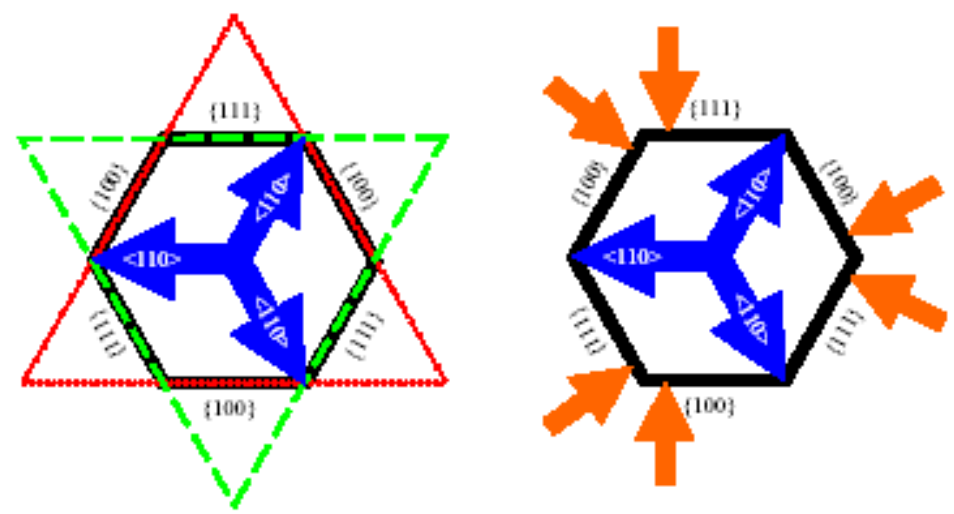

Figure S4. Schematic illustration of the formation of tripod nanocrystals. (Left) In the basal plane of the $\{111\}$ gold nanoplates, the presence of hexagonal shape (black) can be regarded as the favorable structure while the six $<110>$ directions are equivalently extended. Since the side planes of this hexagon are bound alternatively by $\{111\}$ and $\{100\}$ planes, the final shape of the nanoplates is dependent on the competition of growth rates of these two planes. If $\{111\}$ growth is preferentially inhibited, triangular plates (green triangle) are formed; similar condition was met when $\{100\}$ growth is preferentially inhibited (red triangle). (Right) In the case when the growth of the $\{111\}$ and $\{100\}$ planes are simultaneously inhibited, the formation of tripod structures (blue) is highly possible. Despite that the formation of hexapod structures is also a possibility, steric factors may prevent their generation.

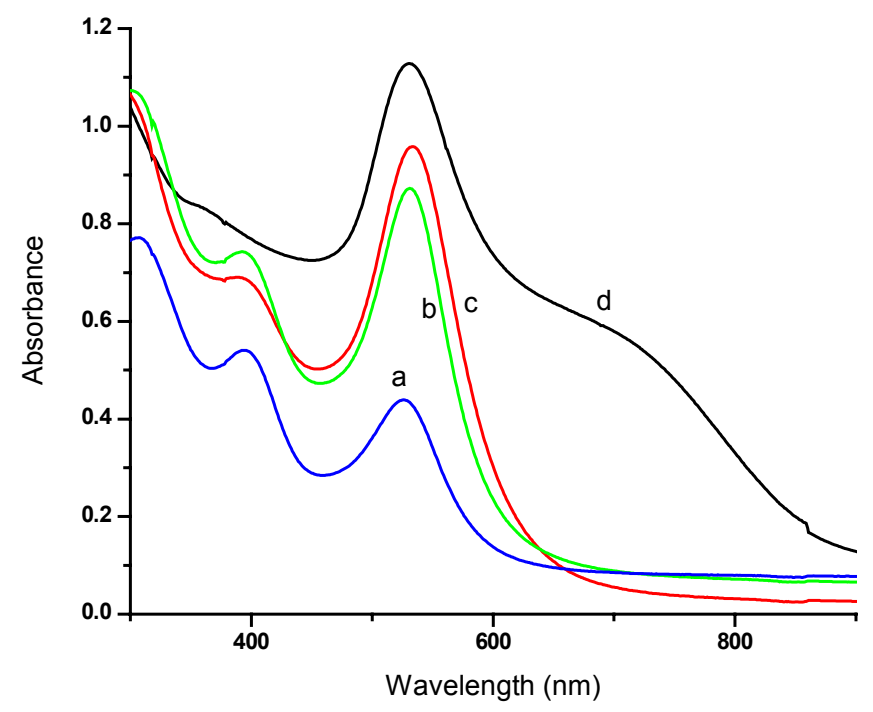

Figure S5. Absorption spectra of the Au particles obtained under otherwise standard conditions at different CTAB concentrations: (a) $0.009 \mathrm{M}$, (b) $0.023 \mathrm{M}$, (c) $0.045 \mathrm{M}$ and (d) $0.090 \mathrm{M}$. The spectra were recorded after 10 min of reactions. Only spherical particles are observed in the cases of (a), (b) and (c), while branched particles are generated in case (d) of the standard condition that is apparent from the existence of a shoulder peak at around $670 \mathrm{~nm}$. 